\title{
Personalized peptide vaccination for castration- resistant prostate cancer progressing after docetaxel: A randomized, double-blind, placebo- controlled, phase III trial
}

Masanori Noguchi ( $\nabla$ noguchi@med.kurume-u.ac.jp )

Showa Daigaku Daigakuin Igaku Kenkyuka Igakubu https://orcid.org/0000-0003-2328-4985

Kiyohide Fujimoto

Nara Medical University Hospital

Gaku Arai

Dokkyo Medical University Saitama Medical Center

Hiroji Uemura

Yokohama City University Medical Center

Katsuyoshi Hashine

Shikoku Cancer Center

Hiroaki Matsumoto

Yamaguchi University Hospital

Satoshi Fukasawa

Chiba Cancer Center

Yasuo Kohjimoto

Wakayama Medical University Hospital

Hideomi Nakatsu

Asahi General Hospital

Atsushi Takenaka

Tottori University Hospital

Masato Fujisawa

Kobe University Hospital

Hirotsugu Uemura

Kinki University Hospital

Seiji Naito

Sanshinkai Hara Hospital

\section{Shin Egawa}

The Jikei University Hospital

Hiroyuki Fujimoto

National Cancer Center Hospital 


\section{Shiro Hinotsu}

Sapporo Medical University

Kyogo Itoh

Kurume University School of Medicine

\section{Research article}

Keywords: Prostate cancer, Cancer vaccine, Multiple-peptide vaccine, Immunotherapy, Docetaxel

Posted Date: February 18th, 2020

DOI: https://doi.org/10.21203/rs.2.23893/v1

License: (c) (i) This work is licensed under a Creative Commons Attribution 4.0 International License. Read Full License 


\section{Abstract}

Background To develop a new treatment modality, we conducted a phase 3 randomized trial of personalized peptide vaccination (PPV) for human leukocyte antigen (HLA)-A24-positive patients with castration-resistant prostate cancer (CRPC) for whom docetaxel chemotherapy failed.

Methods This randomized, double-blind, placebo-controlled, phase 3 trial was done at 68 medical centers in Japan. Eligible patients were aged 20 years or older, positive immunoglobulin $\mathrm{G}(\mathrm{lgG})$ responses to at least 2 of 12 warehouse peptides, an Eastern Cooperative Oncology Group performance status of 0 or 1 , life expectancy of at least 12 weeks, serum testosterone level of $\leq 50 \mathrm{ng} / \mathrm{dl}$, and satisfactory bone marrow function, hepatic function, and renal function. Patients were randomly assigned in a 2:1 ratio to receive PPV or placebo. Four of 12 warehouse peptides selected based on pre-existing peptide-specific IgG levels or the corresponding placebo were subcutaneously injected in 6 doses weekly and then biweekly following the maximum of 30 doses until disease progression. The primary end point was overall survival (OS). Efficacy analyses were by the full analysis set.

Results Between August 2013 and April 2016, 310 patients were randomly assigned, and 306 patients were analyzed. Baseline characteristics were balanced between groups. The estimated median OS was 16.1 months (95\% confidence interval [Cl], 13-18.2) with PPV and 16.9 months $(95 \% \mathrm{Cl}, 13.1-20.4)$ with placebo (hazard ratio [HR], 1.04, 95\% Cl, 0.80-1.37; $p=0.77$ ). Grade $\geq 3$ adverse events were observed in $41 \%$ in both groups. The analysis of treatment arm effects among subgroups revealed lower HRs for OS in favor of the PPV arm in patients with $<64 \%$ neutrophils $(\mathrm{HR}, 0.55,95 \% \mathrm{Cl}, 0.33-0.93 ; \mathrm{p}=0.03)$ or $\geq$ $26 \%$ lymphocytes $(\mathrm{HR}, 0.70,95 \% \mathrm{Cl}, 0.52-0.92 ; \mathrm{p}=0.02)$ at base line.

Conclusions PPV did not prolong OS in HLA-A24-positive patients with CRPC progressing after docetaxel chemotherapy. Subgroup analysis suggested that the patients with a lower proportion of neutrophils or a higher proportion of lymphocytes at base line can receive survival benefits from PPV treatment.

\section{Background}

Since 2004, docetaxel has been the standard first-line chemotherapy for men with metastatic castrationresistant prostate cancer (mCRPC) based on improvements in overall survival (OS) and progression-free survival (PFS) compared with mitoxantrone and prednisone [1, 2]. Although most patients receive docetaxel chemotherapy for MCRPC, the cancer will eventually progress, and no consensus exists for the optimal intervention after docetaxel failure. In recent years, the U.S. Food and Drug Administration (FDA) approved several new drugs for patients with MCRPC for whom docetaxel chemotherapy failed, including cabazitaxel [3], abiraterone with prednisone [4], enzalutamide [5], and radium-223 [6]. Although these advancements have been made, the improvement in survival by these drugs is only several months, and mCRPC continues to be incurable. Thus treatments that can provide durable disease control and longterm survival benefits are needed. 
In the last few decades, immunotherapy has become an important part of treating some types of cancer. Sipuleucel-T is currently the only approved cellular product immune therapy for the treatment of asymptomatic or minimally symptomatic CRPC [7]. Although immune checkpoint inhibitors, including cytotoxic T-lymphocyte antigen 4 (CTLA-4), programed death 1 (PD1), and programed death-ligand 1 (PDL1) were recently approved by the FDA to treat different types of solid tumors and hematologic malignancies, none of these immune checkpoint inhibitors have been approved for MCRPC [8, 9]. In addition, neither peptide-based vaccine trials nor recent conducted immunotherapy studies demonstrated clinical benefits in large randomized trials [10]. This failure may be due to the large diversity of immunological features of MCRPC patients and the lack of readily available biomarkers of immunotherapy benefits.

To overcome these difficulties, we are developing a new concept of personalized peptide vaccination (PPV) for patients with advanced cancer, in which up to 4 peptides are selected from a collection of warehouse peptides based on the pre-existing immunity [11]. A phase I and follow-up study of PPV consisting of 14 warehouse peptides for human leukocyte antigen (HLA) -A24-positive patients with advanced CRPC demonstrated its safety and potential clinical benefit [12]. A randomized phase II trial of PPV with low-dose dexamethasone for patients with chemotherapy-naïve CRPC also resulted in a longer PSA PFS and OS [13]. In addition, another phase II study suggested that the OS of docetaxel-resistant CRPC patients treated with PPV was longer than that of historical controls [14].

Based on these results, a randomized, double-blind, placebo-controlled, phase III trial of PPV for HLA-A24positive patients with CRPC progressing after docetaxel chemotherapy with OS as the primary endpoint was conducted in Japan.

\section{Methods}

\section{Patient population}

For this phase III, randomized, double-blind, placebo-controlled study, we enrolled HLA-A24-positive patients with CRPC progressing within 12 months after docetaxel chemotherapy from 68 medical centers in Japan. Eligible patients were aged 20 years or older with histologically confirmed adenocarcinoma of the prostate. Other inclusion criteria were as follows: positive immunoglobulin $\mathrm{G}(\mathrm{IgG})$ responses to at least 2 of 12 warehouse peptides (Additional file 1) on the screening test, an Eastern Cooperative Oncology Group (ECOG) performance status (PS) of 0 or 1, life expectancy of at least 12 weeks, serum testosterone level of $\leq 50 \mathrm{ng} / \mathrm{dl}$, and satisfactory bone marrow function, hepatic function, and renal function. Patients without previous bilateral orchiectomy continued treatment with luteinizing hormonereleasing agonists. Exclusion criteria included acute infection, history of severe allergic reactions, pulmonary, cardiac or other systemic diseases, or other inappropriate conditions for enrollment as judged by clinicians. Prior enzalutamide or abiraterone was permitted. The trial was conducted in accordance with the Declaration of Helsinki and Good Clinical Practice guidelines. The protocol was approved by institutional review boards or ethical committees at all of the institutions, and it was registered in the 
UMIN Clinical Trials Registry (number: UMIN000011308). All patients were Japanese and provided written informed consent before participating in this study.

\section{Study Design And Treatment}

Patients were randomly assigned in a 2:1 ratio to receive PPV or placebo using the minimization technique with the following stratification factors: age ( $<75$ or $\geq 75)$, PS ( 0 or 1$)$, and use of enzalutamide or abiraterone (with or without) at each participating institution. This study was doubleblinded, and all physicians, patients, and investigators providing the interventions, assessing outcomes, and analyzing data were blinded to treatment assignment. Up to 4 of 12 warehouse peptides selected based on pre-existing peptide-specific IgG levels or the corresponding placebos were emulsified with Montanide ISA 51 incomplete Freund's adjuvant (Seppic, Paris, France), and each study drug (up to 4) in a $1.5-\mathrm{ml}$ emulsion (3 $\mathrm{mg} /$ peptide or saline solution) was subcutaneously injected in 6 doses weekly and then bi-weekly following the maximum of 30 doses until disease progression.

\section{Outcomes}

The primary end point was OS, which was defined as the time from random assignment to death by any cause. Secondary end points were PFS, one-year survival rate, immune responses, and safety. PFS was defined as the time from random assignment until objective disease progression based on the PSA Working Group Consensus Criteria 2 (PCWG2), the Response Evaluation Criteria in Solid Tumors (RECIST) 1.1 criteria, or death. Immune responses were assessed by IgG titers measured by the Luminex system [15] and cytotoxic T lymphocyte (CTL) activity measured by the interferon (IFN) - $\gamma$ release assay [16] using blood sampled at pre-treatment and every 6 treatments. Safety was assessed based on physical examination, vital sign measurements, clinical laboratory analyses, and adverse events (AEs) graded using the Common Terminology Criteria for Adverse Events (CTCAE) version 4.0.

\section{Statistical Analysis}

All efficacy analyses were based on the full analysis set (FAS), defined as patients who had received at least one dose of the treatment. The planned sample size of 300 patients (200 in PPV and 100 in placebo arms) provided $90 \%$ power at a two-sided significance level of 0.05 to detect hazard ratio (HR) of 0.6 for the primary end point of OS, corresponding to an increase in the median OS from 11 to 17 months. All analyses were performed after 23 months from the last patient enrolled or when $80 \%$ of events for the primary end point of OS was reached. The follow-up ended in October 2017, and final analyses were performed using a database frozen in May 2018.

Survival curves were described according to the Kaplan-Meier method and 95\% confidence intervals (Cls) were calculated. The comparison of OS was performed by the Harrington-Fleming test. A stratified logrank test by factors at random assignment was used to compare OS for PPV versus placebo. The effects 
of treatments were reported as $\mathrm{HRs}, 95 \% \mathrm{Cls}$, and interaction $\mathrm{p}$ for subgroup categories in a Forest plot. The mean change from baseline in immune responses at each time point was compared between the two arms in a linear regression model. All toxicity grades and severe (grade $\geq 3$ ) toxicities were compared between the two arms (Fisher's extract test). All statistical tests were interpreted as significant with a pvalue of less than $5 \%$. All analyses were performed using JMP version 13 (SAS Institution, Cary, NC).

\section{Results}

\section{Patient Characteristics}

Between August 2013 and April 2016, 636 patients were screened for eligibility in this study, and 310 eligible patients were randomly assigned to the PPV arm $(n=207)$ or placebo arm $(n=103)$. The most common reason for ineligibility was HLA mismatch. Three patients in the PPV arm and 1 patient in the placebo arm did not receive treatment, and 306 patients were analyzed by the full analysis set (204 for PPV and 102 for placebo). A patient flowchart is shown in Fig. 1. No imbalances existed between randomization arms except for a lower prostate-specific antigen (PSA) level in the PPV arm (Table 1). 
Table 1

Baseline Patient Characteristics by Treatment Arm

Characteristics

Median age, years (range)

Age group (years)

$$
<65
$$

$66-74$

$\geq 75$

ECOG performance status

0

1

Prior use of enzalutamide or abiraterone

yes

no

unknown

Serum PSA level, $\mathrm{ng} / \mathrm{ml}$

$<50$

$50-499$

$\geq 500$

Gleason score at diagnosis

$<6$
7
$\geq 8$
unknown

No. of metastatic sites

0

$<6$

\begin{tabular}{ccc} 
PPV $(n=204)$ & \multicolumn{2}{l}{ Placebo $(n=102)$} \\
No. $\quad \%$ & No. $\quad \%$
\end{tabular}

$71.0(53-84)$

$72.0(56-82)$
37

104

63

162

42

66

44

94

109

83

12

79.4

20.6

32.4

35

34.3

21.6

46

20

47

19.6

46.1

4

2.4

3

2.9

30

14.6

20

19.6

166

80.6

75

73.5

4

2.4

4

4

7.3

7

6.9

Abbreviations; ECOG, Eastern Cooperative Oncology Group; PPV, personalized peptide vaccination; PSA, prostate-specific antigen; WBC, white blood cell. 


\begin{tabular}{|cllll|}
\hline Characteristics & \multicolumn{2}{l}{ PPV $(\mathbf{n}=\mathbf{2 0 4})$} & \multicolumn{2}{l|}{ Placebo $(\mathbf{n}=102)$} \\
\cline { 2 - 5 } & No. & $\%$ & No. & $\%$ \\
\hline 1 & 115 & 56.4 & 61 & 59.8 \\
\hline 2 & 74 & 36.3 & 34 & 33.3 \\
\hline Median proportion of WBC type, \% (range) & & & \\
\hline neutrophils & $70.0(35-94.1)$ & $70.6(39-91)$ \\
\hline Iymphocytes & $21.7(3.6-50.5)$ & $21.4(6-47.1)$ \\
\hline eosinophils & $0.2(0-2)$ & $0.3(0-1.5)$ \\
\hline basophils & $1.0(0-13.8)$ & $1.0(0-11.6)$ \\
\hline monocytes & $6.1(1.7-15)$ & $6.0(2-17)$ \\
\hline Neutrophil to lymphocyte ratio & $3.2(0.7-26.1)$ & $3.3(0.9-15.2)$ \\
\hline $\begin{array}{l}\text { Abbreviations; ECOG, Eastern Cooperative Oncology Group; PPV, personalized peptide vaccination; } \\
\text { PSA, prostate-specific antigen; WBC, white blood cell. }\end{array}$ & \\
\hline
\end{tabular}

\section{Efficacy}

At the final analysis (cut-off date: October 1, 2017), the median length of follow-up for censored patients was 29.8 months in the PPV arm and 27.4 months in the placebo arm, and $89.5 \%$ of patients had discontinued treatment. The median total number of doses of treatment drugs was 12 (IQR, 8 to 19) in the PPV arm and 14 (IQR, 10 to 21) in the placebo arm. The reasons for discontinuation (PPV $\vee$ placebo) were disease progression ( $71.6 \%$ vs $72.5 \%)$, AEs (14.7\% vs $13.7 \%)$, or withdrawal of consent $(3.4 \%$ vs $2.9 \%)$.

For OS, 160 deaths (78.4\%) occurred in the PPV arm and 77 deaths (75.5\%) occurred in the placebo arm. PPV did not improve OS as compared with placebo (median OS, 16.1 months [95\% $\mathrm{Cl}, 13$ to 18.2 months] with PPV and 16.9 months [95\% $\mathrm{Cl}, 13.1$ to 20.4 months] with placebo; $\mathrm{HR}, 1.04 ; 95 \% \mathrm{Cl}, 0.80$ to $1.37 ; \mathrm{p}=$ 0.77; Fig. 2A). The estimated median PFS was 4.2 months ( $95 \% \mathrm{Cl}, 4.0$ to 5.6 months) with PPV and 5.8 months ( $(95 \% \mathrm{Cl}, 4.1$ to 7.3 months) with placebo (HR, $1.09 ; 95 \% \mathrm{Cl}, 0.85$ to $1.40 ; \mathrm{p}=0.32$; Fig. $2 \mathrm{~B})$. The median one-year survival rate was also similar in both arms (62.3\% vs $62.4 \%)$.

For immune responses, pre-treatment plasma and peripheral blood mononuclear cell (PBMC) samples were available for the study from all 306 patients. The mean peptide-specific IgG or CTL levels to the selected peptides were 154 fluorescence intensity units (FIU) or $0.7 \mathrm{pg} / \mathrm{ml}$ in the PPV arm, and 311 FIU or $0.8 \mathrm{pg} / \mathrm{ml}$ in the placebo arm, respectively. After the 6-dose treatment, 189 samples from the PPV arm and 96 samples from the placebo arm were available for immune response analysis, and the mean 
values of IgG and CTL were significantly higher in the PPV arm than in the placebo arm (9716 FIU vs 284 FIU for IgG responses; $p<0.01 ; 80.0 \mathrm{pg} / \mathrm{ml}$ vs $0.7 \mathrm{pg} / \mathrm{ml}$ for CTL activity; $\mathrm{p}<0.01)$. Significant levels of peptide-specific IgG and CTL increase were observed in $27.5 \%$ and $55.6 \%$ in 189 PPV patients, respectively, but no increase was observed in the placebo patients throughout the study period. There was no relationship among IgG, CTL, and OS in the PPV arm by the linear regression model $\left(R^{2}=0.00012\right.$ for IgG change after the 6 -dose treatment; $R^{2}=0.0026$ for $C T L$ change after the 6 -dose treatment).

To further investigate the effects of PPV, we evaluated treatment arm effects among patient subgroups. Initially, we separated subgroups by the subsets listed in Table 1. This analysis revealed lower HRs for OS in favor of the PPV arm in patients with < the median \% neutrophils or $\geq$ the median \% lymphocytes among white blood cell types at baseline (HR, $0.82 ; 95 \% \mathrm{Cl}, 0.56$ to 1.23 for < median \% neutrophil or HR, $0.80 ; 95 \% \mathrm{Cl}, 0.54$ to 1.19 for $\geq$ median \% lymphocyte) by the significant interaction test ( $p=0.04$ or $p=$ 0.05 , respectively; Fig. 3). Given this unexpected interaction among $\%$ neutrophils, $\%$ lymphocytes, and the efficacy of PPV, we analyzed the most relevant \% neutrophil or \% lymphocyte cut-off. We plotted interaction p-values from neutrophil proportions of $50-80 \%$ (median value, $70 \%$ ) or those from lymphocyte proportions of $10-40 \%$ (median value, $21.6 \%$ ) and the number of target patients at each point (Fig. 4). The most relevant \% neutrophil and \% lymphocyte cut-offs were $64 \%$ and $26 \%$, respectively, with an interaction $p<0.01$ and larger number of patients. We reanalyzed treatment arm effects using the cut-off of $64 \%$ neutrophils or $26 \%$ lymphocytes, and found lower HRs for OS in PPV arm patients (HR, $0.55 ; 95 \% \mathrm{Cl}, 0.33$ to 0.93 for $<64 \%$ neutrophils or $\mathrm{HR}, 0.70 ; 95 \% \mathrm{Cl}, 0.52$ to 0.92 for $\geq 26 \%$ lymphocytes) than those in the initial analysis using the median cut-off with an interaction $p=0.003$ or $p=0.007$, respectively (Fig. 3). This interaction among $64 \%$ neutrophils, $26 \%$ lymphocytes, and the PPV treatment is illustrated in Fig. 5. The median OS in the PPV arm of patients with $<64 \%$ neutrophils or $\geq 26 \%$ lymphocytes was significantly longer than median OS in the placebo arm of patients in the same subgroups (median OS, 22.9 vs 15.4 months; $p=0.03$ or median OS, 23.8 vs 13.8 months; $p=0.02$, respectively; Fig. 5A, 5C). In contrast, the median OS in the PPV arm of patients with $\geq 64 \%$ neutrophils or with $<26 \%$ lymphocytes were not different from the median OS in the placebo arm of patients in the same subgroups (Fig. 5B, 5D). On analysis of immune responses for these factors, IgG changes in the PPV arm of patients with $<64 \%$ neutrophils or $\geq 26 \%$ lymphocytes during the first 6 doses were significantly higher than those in patients with in the complementary subgroup $(p=0.02$ or $p=0.01$, respectively; Fig. 5E, 5F), but CTL changes in these subgroups were not significantly different.

All AEs related to the study treatment (peptide plus adjuvant or placebo plus adjuvant) are shown in Table 2. Injection site reactions and nausea were more frequent with PPV ( $86.8 \%$ vs $74.5 \%$ and $9.3 \%$ vs $2.9 \%$, respectively). The most common AEs were < grade 3 injection site reactions in both arms.

Treatment-related AEs of $\geq$ grade 3 were observed in 83 patients in the PPV arm (40.7\%) and 42 patients in the placebo arm (41.2\%). 
Table 2

Any Grade or Severe AEs Recorded in Patients by Treatment Arm (CTCAE version 4.0)

\begin{tabular}{|c|c|c|c|c|c|c|c|c|c|c|}
\hline \multirow[t]{3}{*}{$\mathrm{AE}^{\mathrm{a}}$} & \multirow{2}{*}{\multicolumn{2}{|c|}{$\begin{array}{l}\text { PPV }(n= \\
204) \\
\text { Any grade }\end{array}$}} & \multirow{2}{*}{\multicolumn{2}{|c|}{$\begin{array}{l}\text { Placebo }(n= \\
102) \\
\text { Any grade }\end{array}$}} & \multirow{3}{*}{$\begin{array}{l}p- \\
\text { value } \\
b\end{array}$} & \multirow{2}{*}{\multicolumn{2}{|c|}{$\begin{array}{l}\text { PPV }(n= \\
204) \\
\text { Grade } \geq 3\end{array}$}} & \multirow{2}{*}{\multicolumn{2}{|c|}{$\begin{array}{l}\text { Placebo (n } \\
=102) \\
\text { Grade } \geq 3\end{array}$}} & \multirow{3}{*}{$\begin{array}{l}\text { p- } \\
\text { value } \\
\text { b }\end{array}$} \\
\hline & & & & & & & & & & \\
\hline & No. & $\%$ & No. & $\%$ & & No. & $\%$ & No. & $\%$ & \\
\hline All AEs & 178 & 87.3 & 79 & 77.5 & 0.02 & 83 & 40.7 & 42 & 41.2 & 0.52 \\
\hline $\begin{array}{l}\text { Injection site } \\
\text { reaction }\end{array}$ & 177 & 86.8 & 76 & 74.5 & 0.008 & 5 & 0.0 & 1 & 0.0 & 0.35 \\
\hline Cancer pain & 54 & 26.5 & 22 & 21.6 & 0.21 & 18 & 8.8 & 9 & 8.8 & 0.59 \\
\hline Decreased appetite & 37 & 18.1 & 13 & 12.7 & 0.15 & 19 & 9.3 & 5 & 4.9 & 0.13 \\
\hline Edema peripheral & 35 & 17.2 & 13 & 12.7 & 0.20 & 1 & 0.5 & 2 & 2.0 & 1.0 \\
\hline Pyrexia & 31 & 15.2 & 12 & 11.8 & 0.26 & 2 & 1.0 & 0 & 0.0 & 0.45 \\
\hline Anemia & 29 & 14.2 & 20 & 19.6 & 0.91 & 21 & 10.3 & 12 & 11.8 & 0.72 \\
\hline Constipation & 29 & 14.2 & 14 & 13.7 & 0.53 & 1 & 0.5 & 1 & 1.0 & 0.89 \\
\hline Nasopharyngitis & 28 & 13.7 & 9 & 8.8 & 0.15 & 0 & 0.0 & 0 & 0.0 & n.a. \\
\hline Malaise & 28 & 13.7 & 9 & 8.8 & 0.15 & 2 & 1.0 & 1 & 1.0 & 0.74 \\
\hline Weight gain & 22 & 10.8 & 12 & 11.8 & 0.68 & 2 & 1.0 & 1 & 1.0 & 0.74 \\
\hline Nausea & 19 & 9.3 & 3 & 2.9 & 0.03 & 3 & 1.5 & 1 & 1.0 & 0.59 \\
\hline $\begin{array}{l}\text { Subcutaneous } \\
\text { hemorrhage }\end{array}$ & 19 & 9.3 & 20 & 19.6 & 1.0 & 0 & 0.0 & 0 & 0.0 & n.a. \\
\hline Fall & 17 & 8.3 & 5 & 4.9 & 0.20 & 0 & 0.0 & 1 & 1.0 & 1.0 \\
\hline Diabetes mellitus & 16 & 7.8 & 7 & 6.9 & 0.48 & 4 & 2.0 & 2 & 2.0 & 0.68 \\
\hline Diarrhea & 16 & 7.8 & 8 & 7.8 & 0.60 & 1 & 0.5 & 0 & 0.0 & 0.67 \\
\hline Prostate cancer & 15 & 7.4 & 4 & 3.9 & 0.18 & 15 & 7.4 & 4 & 3.9 & 0.18 \\
\hline Hypertension & 15 & 7.4 & 6 & 5.9 & 0.41 & 10 & 4.9 & 5 & 4.9 & 0.62 \\
\hline Vomiting & 15 & 7.4 & 7 & 6.9 & 0.54 & 1 & 0.5 & 1 & 1.0 & 0.90 \\
\hline
\end{tabular}

Abbreviations: AE, adverse event; CTCAE, Common Terminology Criteria for Adverse Events; n.a., not available; PPV, personalized peptide vaccination.

${ }^{a}$ Medical Dictionary for Regulatory Activities, version 20.0 (MedDRA) terms. ${ }^{\mathrm{b}}$ Fisher's exact test. 


\begin{tabular}{|c|c|c|c|c|c|c|c|c|c|c|}
\hline \multirow[t]{3}{*}{$\mathrm{AE}^{\mathrm{a}}$} & \multirow{2}{*}{\multicolumn{2}{|c|}{$\begin{array}{l}\text { PPV }(n= \\
204) \\
\text { Any grade }\end{array}$}} & \multirow{2}{*}{\multicolumn{2}{|c|}{$\begin{array}{l}\text { Placebo }(n= \\
102) \\
\text { Any grade }\end{array}$}} & \multirow{3}{*}{$\begin{array}{l}p- \\
\text { value } \\
b\end{array}$} & \multirow{2}{*}{\multicolumn{2}{|c|}{$\begin{array}{l}\text { PPV }(n= \\
204) \\
\text { Grade } \geq 3\end{array}$}} & \multirow{2}{*}{\multicolumn{2}{|c|}{$\begin{array}{l}\text { Placebo }(n \\
=102) \\
\text { Grade } \geq 3\end{array}$}} & \multirow{3}{*}{$\begin{array}{l}\text { p- } \\
\text { value } \\
\text { b }\end{array}$} \\
\hline & & & & & & & & & & \\
\hline & No. & $\%$ & No. & $\%$ & & No. & $\%$ & No. & $\%$ & \\
\hline Back pain & 15 & 7.4 & 6 & 5.9 & 0.41 & 2 & 1.0 & 1 & 1.0 & 0.74 \\
\hline Insomnia & 14 & 6.9 & 5 & 4.9 & 0.35 & 0 & 0.0 & 0 & 0.0 & n.a. \\
\hline Weight loss & 12 & 5.9 & 9 & 8.8 & 0.88 & 1 & 0.5 & 1 & 1.0 & 0.90 \\
\hline $\begin{array}{l}\text { Abnormal hepatic } \\
\text { function }\end{array}$ & 11 & 5.4 & 2 & 2.0 & 0.13 & 4 & 2.0 & 0 & 0.0 & 0.20 \\
\hline $\begin{array}{l}\text { Urinary tract } \\
\text { infection }\end{array}$ & 10 & 4.9 & 7 & 6.9 & 0.83 & 3 & 1.5 & 1 & 1.0 & 0.59 \\
\hline Urinary retention & 9 & 4.4 & 6 & 5.9 & 0.92 & 1 & 0.5 & 1 & 1.0 & 0.90 \\
\hline Hydronephrosis & 8 & 3.9 & 7 & 6.9 & 0.92 & 4 & 2.0 & 3 & 2.9 & 0.83 \\
\hline $\begin{array}{l}\text { Platelet count } \\
\text { reduction }\end{array}$ & 8 & 3.9 & 9 & 8.8 & 0.80 & 5 & 2.5 & 2 & 2.0 & 0.57 \\
\hline Hematuria & 7 & 3.4 & 6 & 5.9 & 0.90 & 3 & 1.5 & 2 & 2.0 & 0.79 \\
\hline Dental caries & 3 & 1.5 & 6 & 5.9 & 0.99 & 1 & 0.5 & 0 & 0.0 & 0.67 \\
\hline \multicolumn{11}{|c|}{$\begin{array}{l}\text { Abbreviations: AE, adverse event; CTCAE, Common Terminology Criteria for Adverse Events; n.a., not } \\
\text { available; PPV, personalized peptide vaccination. }\end{array}$} \\
\hline
\end{tabular}

\section{Discussion}

PPV was demonstrated in previous phase II trials to improve OS in chemotherapy-naïve patients with CRPC or in patients with docetaxel-resistant CRPC. This phase III randomized trial investigated PPV as a second-line treatment after docetaxel chemotherapy in patients with progressing CRPC. To our knowledge, this is the first trial addressing this strategy in a relatively large number of patients for whom docetaxel induction chemotherapy failed. In this trial, no difference in OS or PFS between PPV and placebo was found.

There are several explanations for the lack of OS improvement in this study. First, target patients may have heterogeneous immune cell repertoires, a large tumor burden, and many immune suppressive elements, such as increased myeloid-derived suppressor cells (MDSC) or regulatory T cells (Treg) in the tumor microenvironment, and it is well known that tumor-associated immunosuppression is significantly involved in tumor progression and resistance to immunotherapy $[17,18]$. Second, another contributory 
factor may be the availability of more effective salvage therapies that prolong OS after the study treatment, many of which were not widely available at the time of the previous phase II study. The current availability of such drugs (eg, cabazitaxel, abiraterone, enzalutamide, and radium-223) may have affected the disease course in patients receiving PPV or placebo. Indeed, 148 of 204 patients $(72.5 \%)$ in the PPV arm and 71 of 102 patients (69.6\%) in the placebo arm used such drugs after the study treatment. Third, the treatment discontinued early before sufficient doses of PPV were administered, thereby affecting the efficacy due to the lack of notable objective responses or PSA progression. The median dose of PPV was 12 (planned dose was up to 36), and only 21 of 204 patients (10.3\%) completed the treatment in the PPV arm.

Peptide based vaccines have been designed to elicit CTL against antigens selectively expressed by tumor cells. PPV, in which a maximum of four HLA class IA-matched peptides are selected for vaccination from a pool of peptides on the basis of both HLA class IA type and the preexisting host immunity before vaccination, are designed to stimulate antigen-specific memory T cells. In a trial of neoadjuvant peptide vaccination before radical prostatectomy for patients with localized prostate cancer, we previously reported that PPV quickly induced infiltration of CD45RO + memory T cells, rather than naïve T cells or B cells, into cancer tissues [19]. We evaluated the preexisting immunity to vaccine candidate by peptidespecific IgG responses in pre-vaccination plasma, since our previous clinical trials demonstrated that the IgG-based selection is useful for predicting CTL boosting after vaccination $[12,20]$. Our previous study also suggested the significance of peptide-specific IgG responses in patients with advanced cancer treated by PPV as a surrogate biomarker in monitoring vaccine-induced immune and clinical responses [21]. In this trial, there was no relationship among IgG changes, CTL changes, and OS in the PPV arm. However, IgG changes in the PPV arm of patients with $<64 \%$ neutrophils or $\geq 26 \%$ lymphocytes who showed a long OS, were significantly higher than those in patients with the complementary subgroup.

In general, the development of peptide-based vaccines has some issues to be solved. Peptides used in peptide-based vaccines are restricted by HLA class I or II molecule, and they have limitations when used in clinical trials. In addition, tumor cells, can downregulate the expression of these molecules. In this clinical trial, we used HLA-A24-restricted peptides, which are the most common in Japanese because they are targeted at Japanese. Another issue is that efficiently primed $T$ cells often lose their responsiveness to tumor antigens. This may be explained by down-regulation or loss of tumor antigens, immunosuppression by Treg and MDSC cells, and T cell inhibition mediated by checkpoint molecules, such as CTLA-4, PD-1, and PDL-1 [22, 23]. Although we did not evaluate these mechanisms in this study, our previous analysis to evaluate effect of 20-mixed peptides for patients with CRPC showed that no obvious difference were observed in the frequency of Treg and MDSC and the level of serum CTLA-4 during the vaccination [24].

Further study of predictive biomarkers of PPV efficacy may be warranted to determine whether subgroups will improve the OS. The recent discovery that cancers deficient in DNA mismatch-repair function (dMMR) or with microsatellite instability (MSI-high) demonstrate high rates of objective tumor responses to immune checkpoint therapies [25] led to the FDA approval of pembrolizumab for the treatment of

Page $12 / 23$ 
advanced dMMR/MSH-high cancers of any histologic type, among which mCRPC patients are a very small subset. We recently reported that the abnormal granulocytes present in the PBMC fraction at baseline may lead to the poor prognosis of advanced prostate cancer patients receiving PPV treatment using DNA microarray analysis [26]. We also demonstrated that the increase in granulocytic MDSC after PPV treatment was an unfavorable marker for the OS of mCRPC patients [27]. This suggested that the proportion of neutrophils, the majority of granulocytes, affects the efficacy of PPV treatment. The post hoc analysis in this trial demonstrated that patients with a low neutrophil proportion ( $<4 \%)$ or a high lymphocyte proportion ( $\geq 26 \%$ ) at baseline in the PPV arm had a significantly longer OS than their counterparts in the placebo arm; however, the proportions of eosinophils, basophils, and monocytes did not affect the efficacy of PPV treatment even though the neutrophil to lymphocyte ratio (NLR) was used. Although NLR was reported as a risk factor for the OS of patients with MCRPC as well as many other advanced cancers when it was higher than 2 [28-30], we found that the NLR was less sensitive than the proportion of neutrophils or lymphocytes as a biomarker to predict the efficacy of PPV with an interaction $p=0.09$. This discrepancy in results may have occurred, in part, because the NLR value as a risk factor was based on a comparison of the lower and higher levels among patients in the same treatment arm in the previous studies rather than between patients in different treatment arms (i.e., PPV and placebo). The levels of PPV-induced IgG were significantly higher in patients with $<64 \%$ neutrophils or $\geq 26 \%$ lymphocytes treated by PPV than in their counterparts, and the median OS of these groups was significantly longer than that of the counter groups. These results suggested that patients with $<64 \%$ neutrophils or $\geq 26 \%$ lymphocytes can receive survival benefits from PPV treatment. Although further validation is required, this hypothesis is novel and helpful to prolong survival in patients with CRPC progressing after docetaxel chemotherapy.

The tolerability of PPV treatment was good overall, and the most common treatment-related AE in both arms was injection site reaction, which was mainly caused by incomplete Freund' adjuvant [31]. Dose interruptions or reductions were infrequent, and the overall safety profile was consistent with that observed in previous phase II trials. Treatment-related deaths were not increased with PPV, suggesting a lack of toxicity as the main contributing factor.

In conclusion, PPV did not prolong OS or PFS in HLA-A24-positive patients with CRPC progressing after docetaxel chemotherapy. Subgroup analyses demonstrated that patients with a low neutrophil proportion or a high lymphocyte proportion at baseline in the PPV arm had a significantly longer OS than their counterparts in the placebo arm in this setting. Additional validation of this finding is needed to better define subgroups of patients who can receive PPV treatment for progressing CRPC after docetaxel chemotherapy.

\section{Abbreviations}

AEs: adverse events; CRPC: castration-resistant prostate cancer; CTL: cytotoxic T lymphocytes; ECOG: Eastern Cooperative Oncology Group; HLA: human leukocyte antigen; IgG: immunoglobulin G; MDSC: myeloid-derived suppressor cells; NLR: neutrophil to lymphocyte ratio; OS, overall survival; PD: 
progressive disease; PFS: progression-free survival; PPV: personalized peptide vaccination; PSA: prostatespecific antigen; Treg, regulatory $T$ cells

\section{Declarations}

\section{Acknowledgement}

The authors thank all the investigators, study coordinators, and nurses within the participating centers. The authors also acknowledge all the patients and their families.

\section{Declarations}

\section{Author's contribution}

$\mathrm{MN}, \mathrm{HU}, \mathrm{SN}, \mathrm{SE}, \mathrm{HF}$, and $\mathrm{KI}$ contributed to the conception and design of the study. conceived the study. KF, $\mathrm{GA}, \mathrm{HU}, \mathrm{KH}, \mathrm{HM}, \mathrm{SF}, \mathrm{YK}, \mathrm{HN}, \mathrm{AT}, \mathrm{MF}, \mathrm{SN}, \mathrm{SE}$, and HF provided study materials or patients. MN and SH completed the statistical analyses. MN reviewed the clinical data. All authors interpreted data and reviewed and approved the final article.

\section{Funding}

This study was supported by grants from the Japan Agency for Medical Research and Development (No. 18im0110802h0008) and Fujifilm Company. The funders of the study had no role in the study design, data collection, data analysis, data interpretation, or writing of the report.

\section{Availability of data and materials}

The datasets used and/or analyzed during the current study are available from the corresponding author on reasonable request.

\section{Ethics approval and consent to participate}

This study was approved by the Ethical Committee of Kurume University (approval No. 213019) and each institution, and it was registered in the UMIN Clinical Trials Registry (number: UMIN000011308) and the Pharmaceuticals and Medical Devices Agency in Japan (number: 25-0917). The study was carried out in accordance with the Declaration of Helsinki and the International Conference on Harmonization of Good Clinical Practice guidelines. Written-informed consent to participate in the clinical trial and to use their 
data for research and publication purpose was received from all individual participants before participating in the study.

\section{Consent for publication}

Not applicable.

\section{Competing interests}

MN has served as an advisory board consultant for BrightPath Biotherapeutics Co. Ltd. KI received research funding from Taiho Pharmaceutical Company. SN has served as a consultant to BrightPath Biotherapeutics Co. Ltd and received an honorarium from Sanofi. All other authors declare no competing interests.

\section{References}

1. Tannock IF, de Wit R, Berry WR, Horti J, Pluzanska A, Chi KN, et al. Docetaxel plus prednisone or mitoxantrone plus prednisone for advanced prostate cancer. N Engl J Med. 2004;351:1502-12.

2. Berthold DR, Pond GR, Soban F, de Wit R, Eisenberger M, Tannock IF. Docetaxel plus prednisone or mitoxantrone plus for advanced prostate cancer: updated survival in the TAX 327 study. J Clin Oncol. 2008;26:242-5.

3. De Bono JS, Oudard S, Ozgurogh M, Hansen S, Machiels JP, Kocak I, et al. Prednisone plus cabazitaxel or mitoxantrone for metastatic castration-resistant prostate cancer progressing after docetaxel treatment: a randomized open-label trial. 2010;376:1147-54.

4. De Bono JS, Logothetis CJ, Molina A, Fizazi K, North S, Chu L, et al. Abiraterone and increased survival in mPrCa. N Engl J Med. 2011;354:1995-2005.

5. Scher HI, Fizazi K, Sadd F, Taplin ME, Sternberg CN, Miller K, et al. Increased survival with enzalutamide in prostate cancer after chemotherapy. N Engl J Med. 2012;367: 1187-97.

6. Parker C, Nilsson S, Heinrich D, Helle SI, O'Sullivan JM, Fosså SD, et al. Alpha emitter radium-223 and survival in metastatic prostate cancer. N Engl J Med. 2013;369:213-23.

7. Kantoff PW, Higano CS, Shore ND, Berger ER, Small EJ, Penson DF, et al. Sipuleucel-T immunotherapy for castration-resistant prostate cancer. N Engl J Med. 2010;363:411-22.

8. Issacsson VP, Antonarakis ES. PD-1/PD-L1 pathway inhibitors in advanced prostate cancer. Expert Rev Clin Pharmacol. 2018;11:475-86.

9. Kwon ED, Drake CG, Scher HI, Fizazi K, Bossi A, van den Eertwegh AJ, et al. Ipilimumab versus placebo after radiotherapy in patients with metastatic castration-resistant prostate cancer that had 
progressed after docetaxel chemotherapy (CA184-043): a randomized double-blind, phase 3 trial. Lancet Oncol. 2014;15:700-12.

10. Nuhn P, De Bono JS, Fizazi K, Freedland SJ, Grilli M, Kantoff PW, et al. Update on systemic prostate cancer therapies: management of metastatic castration-resistant prostate cancer in the era of precision oncology. Eur Urol. 2019;75:88-99.

11. Noguchi M, Sasada T, Itoh K. Personalized peptide vaccination: a new approach for advanced cancer as therapeutic cancer vaccine. Cancer Immunol Immunother. 2013;62:919-29.

12. Noguchi M, Uemura H, Naito S, Akaza H, Yamada A, Itoh K. A phase I study of personalized peptide vaccination using 14 kinds of vaccine in combination with low-dose estramustine in HLA-A24positive patients with castration-resistant prostate cancer. 2011;71:470-9.

13. Yoshimura K, Minami T, Nozawa M, Kimura T, Egawa S, Fujimoto H, et al. A phase 2 randomized controlled trial of personalized peptide vaccine immunotherapy with low-dose dexamethasone versus dexamethasone alone in chemotherapy-naïve castration-resistant prostate cancer. Eur Urol. 2016;70:35-41.

14. Noguchi M, Moriya F, Suekane S, Matsuoka K, Arai G, Matsueda S, et al. Phase II study of personalized peptide vaccination for castration-resistant prostate cancer patients who failed in docetaxel-based chemotherapy. 2012;72:834-45.

15. Komatsu N, Shichijo S, Nakagawa M, Itoh K. New multiplexed flow cytometric assay to measure antipeptide antibody: a novel tool for monitoring immune responses to peptides used for immunization. Scand J Clin Lab Invest. 2004; 64:535-45.

16. Hida N, Maeda Y, Katagiri K, Takasu H, Harada M, Itoh K. A simple culture protocol to detect peptidespecific cytotoxic T lymphocyte precursors in the circulation. Cancer Immunol Immunother. 2002;51:219-28.

17. Gabrilovich DI, Bronte V, Chen SH, Colombo MP, Ochoa A, Ostrand-Rosenberg S, et al. The terminology issue for myeloid-derived suppressor cells. Cancer Res. 2007; 67:425-6.

18. Miller AM, Lundberg K, Ozenci V, Banham AH, Heilsyrom M, Egevad I, et al. CD4 ${ }^{+} C D 25$ high T cells are enriched in the tumor and peripheral blood of prostate cancer patients. J Immunol. 2006;177:7398-

19. Noguchi M, Yao A, Harada M, Nakashima O, Komohara Y, Yamada S, et al. Immunological evaluation of neoadjuvant peptide vaccination before radical prostatectomy for patients with prostate cancer. 2007;67:933-42.

20. Terasaki M, Shibui S, Narita Y, Fujimaki T, Aoki T, Kajiwara K, et al. Phase I trial of a personalized peptide vaccine for patients positive for human leukocyte antigen-A24 with recurrent or progressive glioblastoma multiforme. J Clin Oncol. 2011;29:337-44.

21. Noguchi M, Mine T, Komatsu N, Suekane S, Moriya F, Matsuoka K, et al. Assessment of immunological biomarkers in patients with advanced cancer treated by personalized peptide vaccination. Cancer Biol Ther. 2011;10:1266-79.

22. Topalian SL, Hodi FS, Brahmer JR, Gettinger SN, Smith DC, McDermott DF, et al. Safety, activity, and immune correlates of anti-PD-1 antibody in cancer. N Engl J Med. 2012;366:2443-54. 
23. Andeerson MJ, Shafer-Weaver K, Greenberg NM, Hurwitz AA. Tolerization of tumor-specific T cells despite efficient initial priming in a primary murine model of prostate cancer. $J$ Immunol. 2007;178:1268-76.

24. Noguchi M, Arai G, Matsumoto K, Naito S, Moriya F, Suekane S, et al. Phase I trial of a cancer vaccine consisting of 20 mixed peptides in patients with castration-resistant prostate cancer: dose-related immune boosting and suppression. Cancer Immunol Immunother. 2015;64:493-505.

25. Le DT, Durham JN, Smith KN, Wang H, Bartlett BR, Aulakh LK, et al. Mismatch repair deficiency predicts response of solid tumors to PD-1 blockade. 2017;357:409-13.

26. Komatsu N, Matsueda S, Tashiro K, loji T, Shichijo S, Noguchi M, et al. Gene expression profiles in peripheral blood as a biomarker in cancer patients receiving peptide vaccination. 2012;118:3208-21.

27. Noguchi M, Moriya F, Koga N, Matsueda S, Sasada T, Yamada A, et al. A randomized phase II clinical trial of personalized peptide vaccination with metronomic low-dose cyclophosphamide in patients with metastatic castration-resistant prostate cancer. Cancer Immunol Immunother. 2016;65:151-60.

28. Coffelt SB, Wellenstein MD, de Visser KE. Neutrophils in cancer: neutral no more. Nat Rev Cancer. 2016;16:431-46.

29. Shaul ME, Fridlender ZG. Cancer-related circulating and tumor-associated neutrophils - subtypes, sources and function. FEBS J. 2018;285:4316-42.

30. Dolan RD, Laird BAJ, Horgan PG, McMillan DC. The prognostic value of the systemic inflammatory response in randomized clinical trials in cancer: A systematic review. Critical Reviews in Oncology / Hematology. 2018;132:130-7.

31. van Doorn E, Liu H, Huckriede A, Hak E. Safety and tolerability evaluation of the use of Montanide ISATM51 as vaccine adjuvant: A systematic review. Hum Vaccin Immunother. 2016;12:159-69.

\section{Figures}




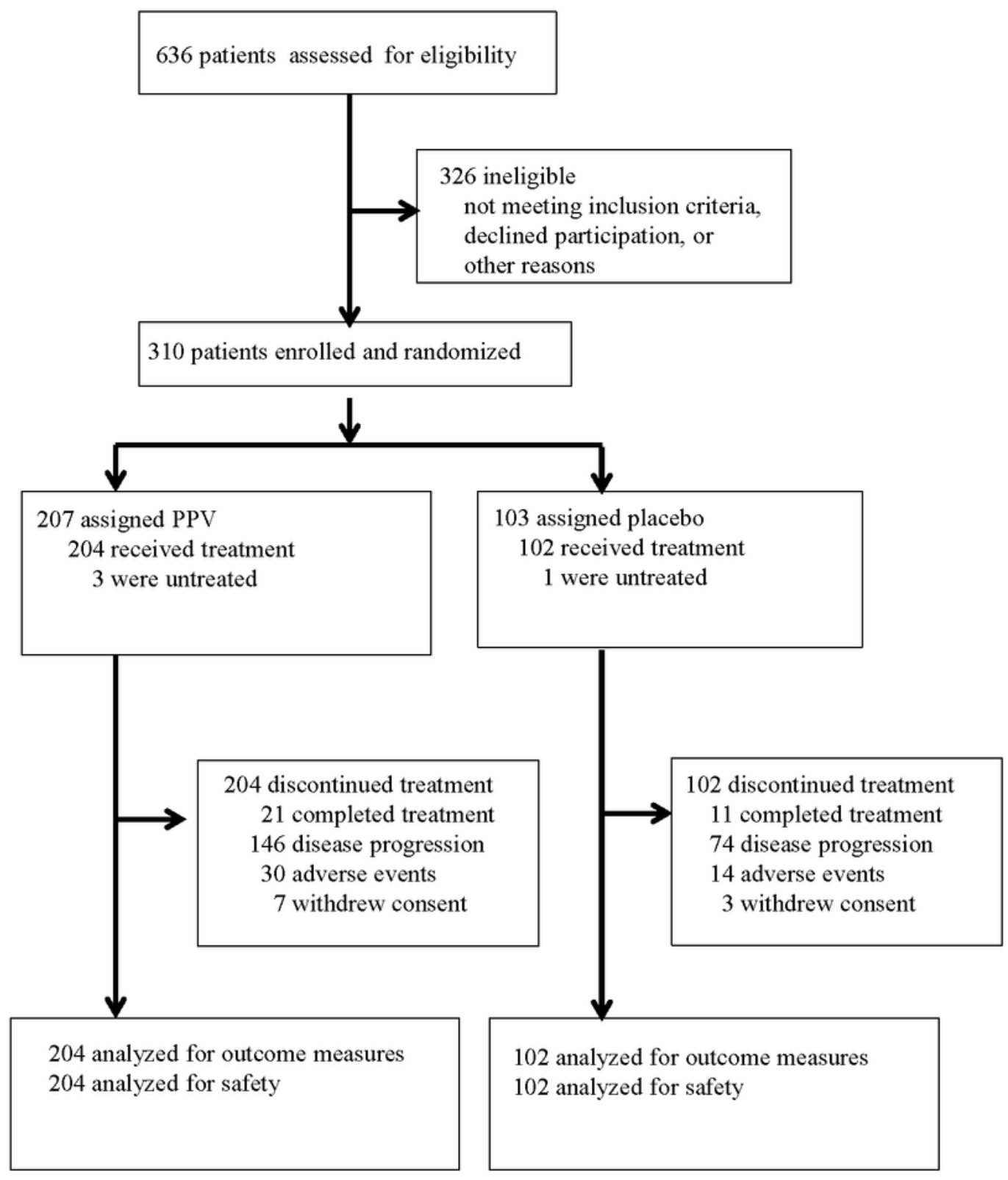

Figure 1

CONSORT diagram. PPV, personalized peptide vaccination. 
A

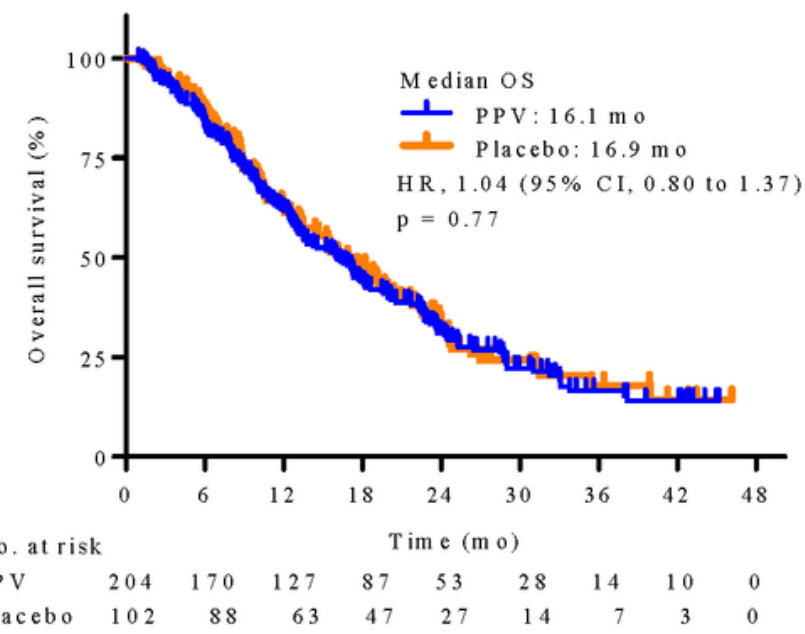

B

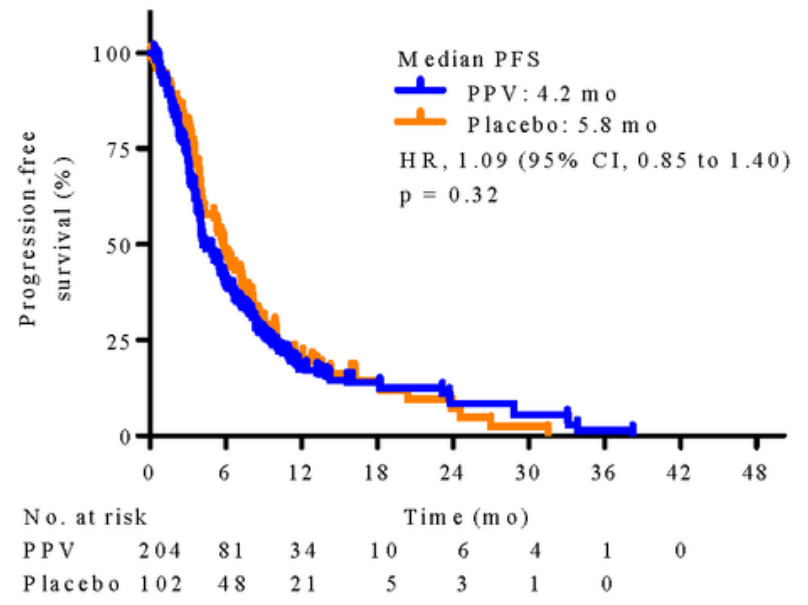

\section{Figure 2}

Kaplan-Meier curves according to study treatment. (A) Progression-free survival. (B) Overall survival. 


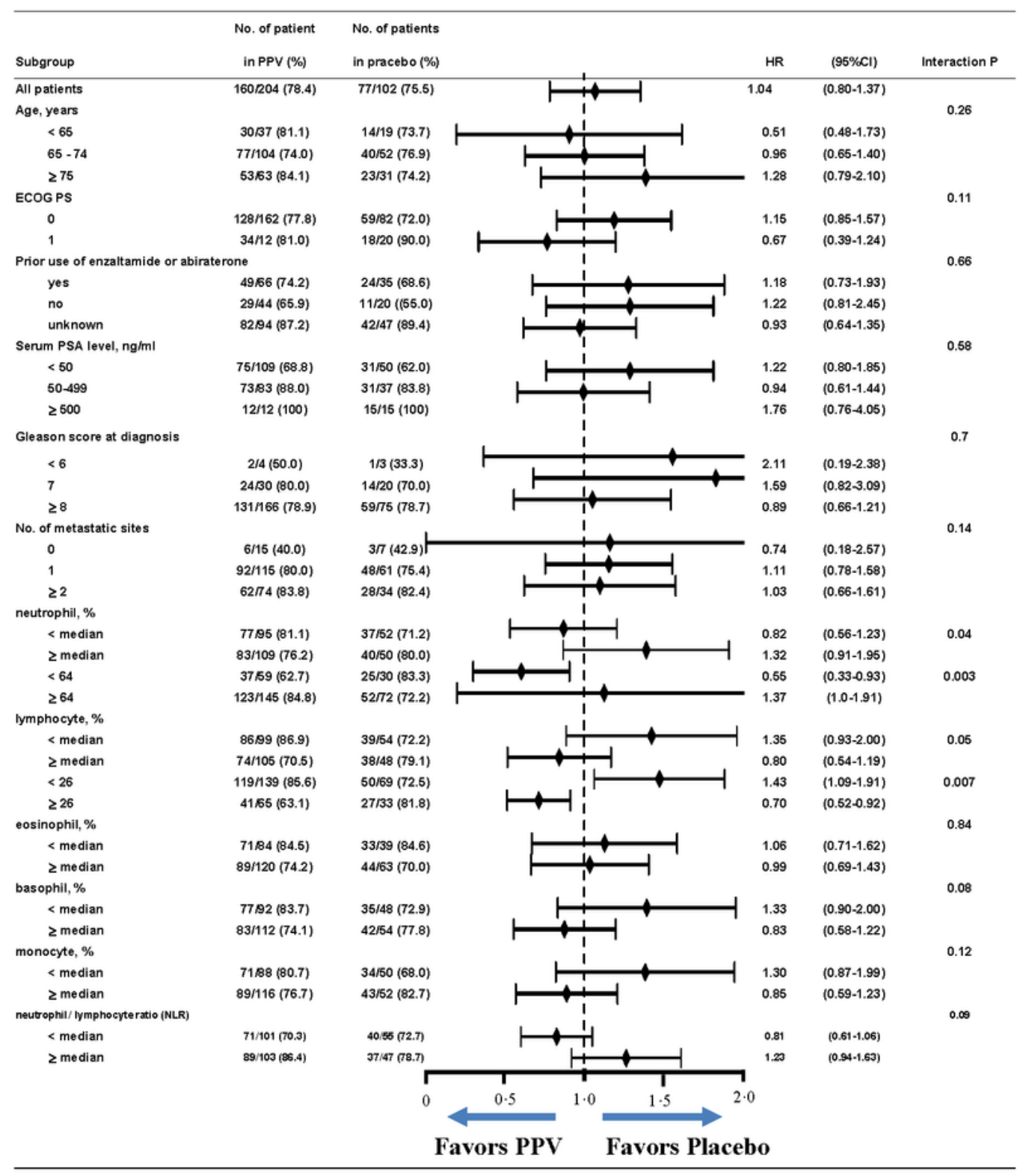

\section{Figure 3}

Forest plot for subgroup analysis of stratification factors (full analysis set population). ECOG, Eastern Cooperative Oncology Group; HR, hazard ratio; PPV, personalized peptide vaccination; PS, performance status; PSA, prostate-specific antigen. 
A

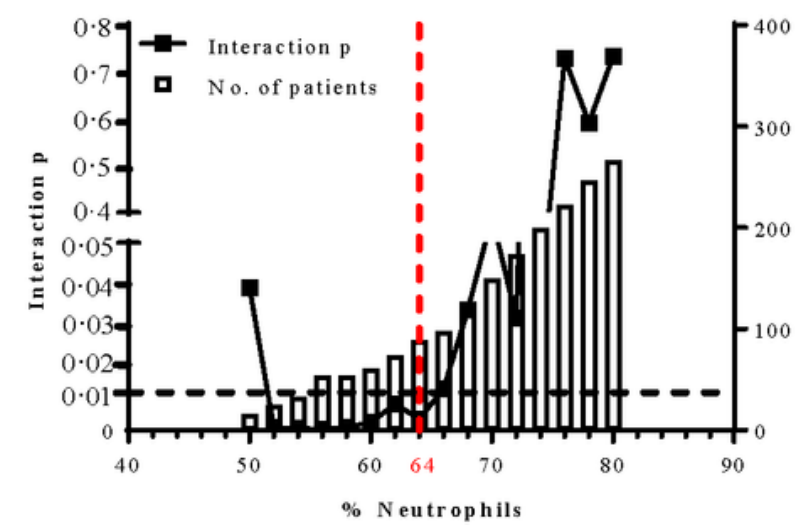

B

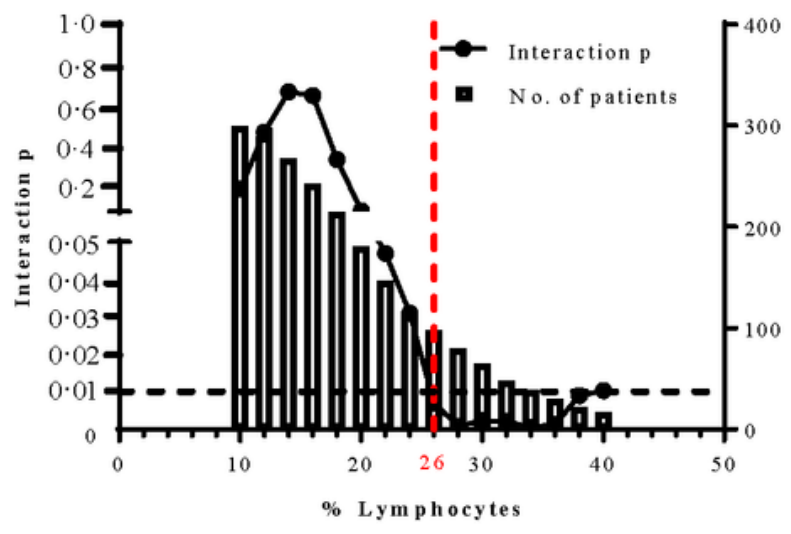

Figure 4

The most relevant \% neutrophil or \% lymphocyte cut-off. Relationship among \% neutrophils (A), \% lymphocytes (B), interaction $\mathrm{P}$, and number of patients. 
A

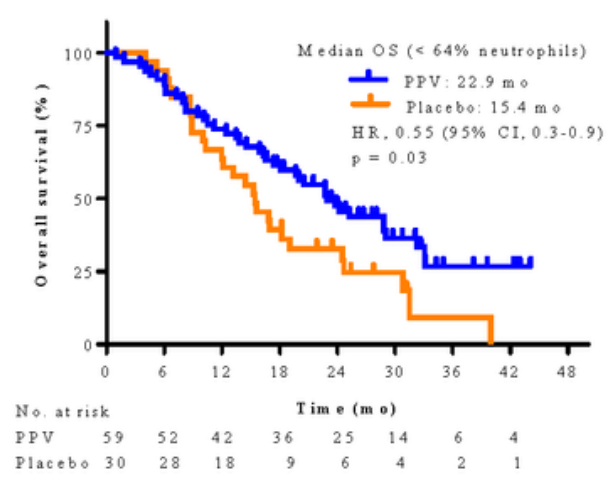

C

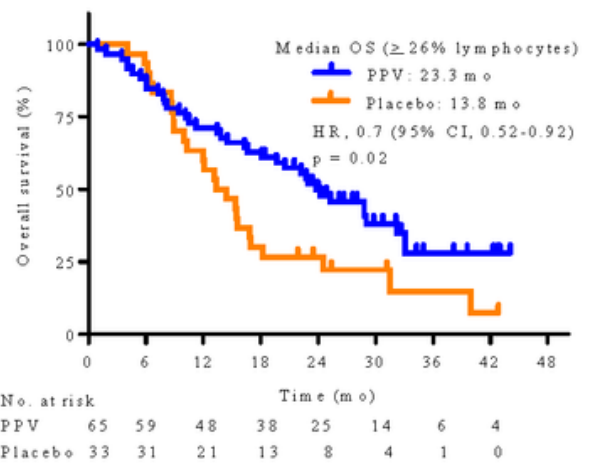

E

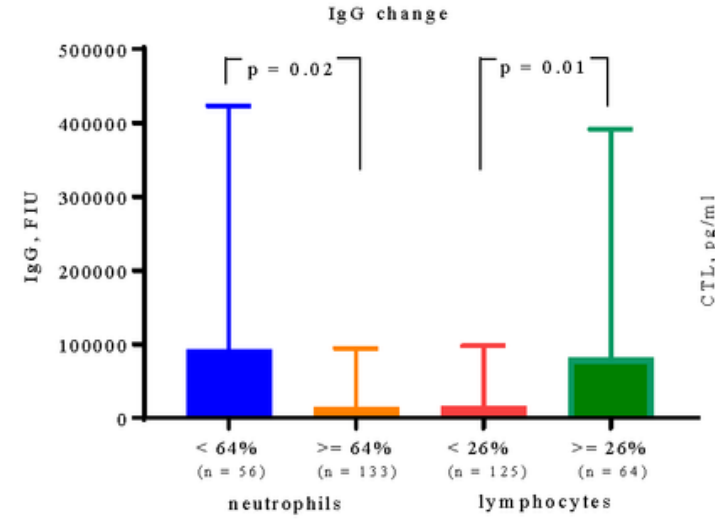

B

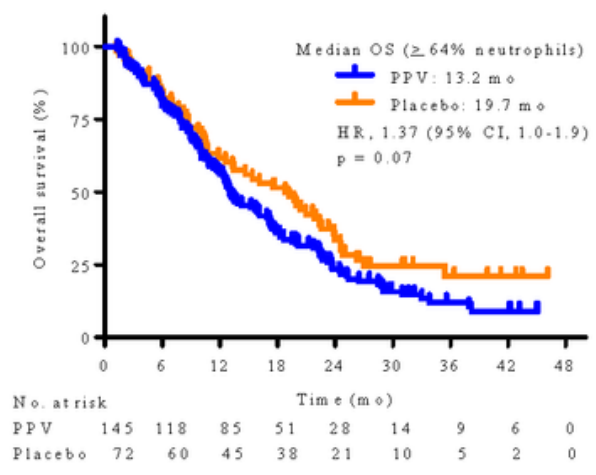

D

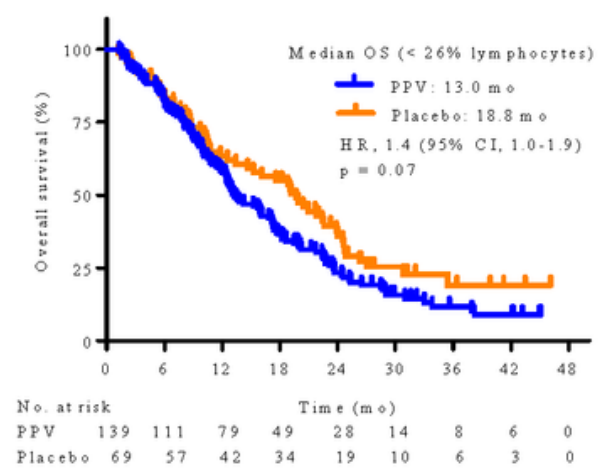

F

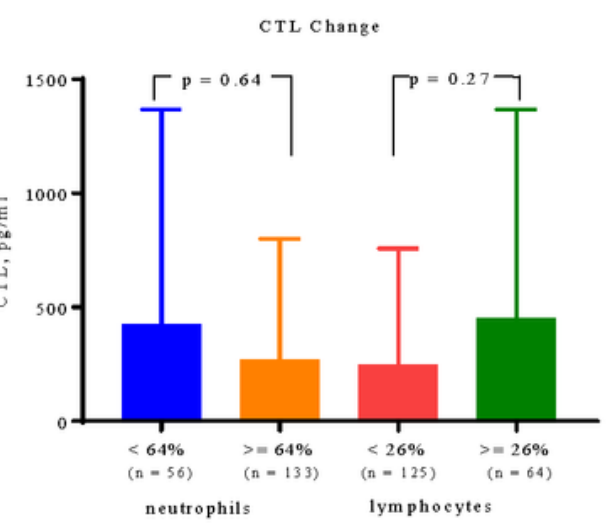

\section{Figure 5}

Comparison of treatment effects. (A) Overall survival (OS) in patients with $<64 \%$ neutrophils according to personalized peptide vaccination (PPV) versus placebo treatment. (B) OS in patients with $\geq 64 \%$ neutrophils according to PPV versus placebo treatment. (C) OS in patients with $\geq 26 \%$ lymphocytes according to PPV versus placebo treatment. (D) OS in patients with $<26 \%$ lymphocytes according to PPV versus placebo treatment. (E) Changes in IgG or CTL levels from baseline to 6 doses in the PPV arm 
according to neutrophil proportion ( $<64 \%$ or $\geq 64 \%$ ). (F) Changes in IgG or CTL levels from baseline to 6 doses in the PPV arm according to neutrophil proportion ( $\geq 26 \%$ or $<26 \%$ ). CTL, cytotoxic T lymphocyte; FIU, fluorescence intensity units; IgG, immunoglobulin G; HR, hazard ratio.

\section{Supplementary Files}

This is a list of supplementary files associated with this preprint. Click to download.

- Additionalfile1.docx 